\title{
Photoelectrochemical spectroscopy studies of titanium dioxide surfaces: theory and experiment
}

\author{
J.W. Halley ${ }^{\text {a,d }}$, M. Kozlowski ${ }^{\text {b,d }}$, M. Michalewicz ${ }^{\text {a,c,d }}$, W. Smyrl ${ }^{\text {d }}$ and N. Tit ${ }^{\text {a,d }}$ \\ "School of Physics and Astronomy, University of Minnesota, Minneapolis, MN 55455, USA \\ ${ }^{b}$ Lawrence Livermore National Laboratory, Livermore, CA 94550, USA \\ 'Commonwealth Scientific and Industrial Research Organization, Division of Information Technology, 55 Barry St., Carleton, \\ Victoria, Australia \\ 'Corrosion Research Center, Department of Chemical Engineering and Materials Science, University of Minnesota, Minneapolis, \\ MN 55455, USA
}

Received 17 December 1990; accepted for publication 22 April 1991

\begin{abstract}
We describe experimental and theoretical studies of titanium dioxide. Photoelectrochemical data are presented for surfaces of known structure. The oxides were grown anodically on titanium metal. We report calculations of the electronic densities of states and optical conductivities of rutile $\mathrm{TiO}_{2}$ in finite-size samples disordered by the addition of oxygen vacancies for comparison with experiments. We conclude that in less disordered surfaces the electronic structure exhibited by the photospectra is very close to that of bulk rutile $\mathrm{TiO}_{2}$. In contrast, very thin oxides showed evidence of an electronic structure dominated by localized states near the band edge. More disordered oxides gave photospectra in which transitions associated with direct band gap transitions in bulk $\mathrm{TiO}_{2}$ seem to have been suppressed. Taking the structural information on the oxides into account, we argue that this suppression is probably a matrix element effect.
\end{abstract}

\section{Introduction}

Several studies of the photoelectrochemical properties of the surface of anodic oxides on titanium have been reported [1-5]. In these studies the authors evaluated the photoelectrochemical spectra of the oxide using the Gaertner--Butler model (based on the assumption of parabolic band structure) for semiconductor/electrolyte junctions [6]. Estimations of the optical bandgaps were made and conclusions regarding the oxide crystal structure and morphology were drawn. Indirect bandgaps of $3.3 \mathrm{eV}$ were typically reported for the oxides [2,3]. This value is higher than the lowest bandgaps characteristic of single-crystal rutile ( 3.0 $\mathrm{eV})$ and anatase $(3.2 \mathrm{eV})$. In this earlier work the high bandgap of the anodic oxides was attributed to changes in the electronic structure associated with the disordered nature of the oxides. In ref. [1] no direct bandgaps were reported for thin oxides grown to less than $15 \mathrm{~V}$ (SCE) and this led the investigators to conclude that the oxides were amorphous. The appearance of the direct bandgap for thicker oxides was associated with a recrystallization of the oxide. In studies of the photocurrent-bias characteristics, McAleer and Peter [5] observed that only oxides grown slowly, by ramping the electrode potential at rates below $5 \mathrm{mV} / \mathrm{s}$, behaved as predicted by the Gaertner-Butler model for ideal materials. They attributed the deviations observed for more rapidly grown oxides to a disordered structure. Leitner et al. [1] reported that the near-band edge spectral response of oxides grown at high anodic currents (14 $\mathrm{mA} / \mathrm{cm}^{2}$ ) followed an "Urbach tail" exponential dependence on energy [7] associated with localized states in the bandgap and characteristic of highly disordered semiconductors. Leitner et al. [1] also showed that in some cases the current-voltage characteristics of the oxides follow the PooleFrenkel dependence [8] associated with charge transport in disordered materials. In these studies, 
no structural measurements were made to support the investigators' conclusions regarding the oxide structure.

In a previous paper [9], we reported photoelectrochemical spectra of anodic oxides grown on polycrystalline titanium substrates. The results were directly correlated with electron diffraction measurements made on surfaces prepared under identical conditions. The electron diffraction studies showed that the oxides of interest always had a rutile structure. The size and degree of ordering of oxide grains was dependent on the oxide growth rate and substrate crystallography. Oxides grown by potential ramping at $0.1 \mathrm{mV} / \mathrm{s}$, the lowest growth rate reported in the literature, showed preferred growth orientations and in some cases partial epitaxy, depending on the substrate grain orientation. As the growth rate was increased the oxides become homogeneous and more disordered. In ref. [9] we used the assumption of parabolic bands to make a photoelectrochemical analysis of the oxides. The analysis suggested an indirect bandgap of $3.3 \mathrm{eV}$ and a direct bandgap of $3.7 \mathrm{eV}$ for slowly grown oxides. For rapidly grown oxides, no evidence of a direct bandgap emerged from the analysis. Correlation of the structural and photoelectrochemical data showed that the oxides which showed no evidence of a direct bandgap were not necessarily amorphous. Evidence of a high indirect bandgap was observed even for highly ordered epitaxial rutile oxides.

In other earlier work [10] we reported the calculation of the electronic structure of rutile $\mathrm{TiO}_{2}$ containing oxygen vacancies. The calculation used extensions of the full tight-binding model of Vos [11] to study the density of states as a function of oxygen vacancy concentration and film thickness. The calculations provided details concerning the nature of band gap states and tails arising from oxygen vacancies and showed that finite thickness of the oxide affects the electronic structure only for very thin ( $\leq 1.0 \mathrm{~nm}$ ) oxides.

In the present paper, we apply the theoretical model of ref. [10] to the interpretation of experiments partly reported in ref. [9]. In the case of thick, slowly grown oxides, we will show in this paper that the electronic structure is much closer to that of bulk rutile than was previously believed.
We establish this both by comparison of our results with experimental photospectra of bulk $\mathrm{TiO}_{2}$ and also by comparison between our experimental photospectra and predicted photospectra which we calculate from extensions of the tight-binding model of Vos for $\mathrm{TiO}_{2}$. For very thin oxides and for oxides grown at high rates, the electronic structure deviates further from that of singlecrystal rutile. We discuss possible theoretical explanations of these results as well.

In the next two sections we report our experimental and theoretical methods. Sections four and five summarize the experimental and theoretical results and the last section summarizes our conclusions. The last section includes a discussion of the effects of the surface potential and the possible effects of self-consistency and localization on the calculations.

\section{Experimental methods}

Anodic oxides were grown on etched polycrystalline titanium substrates as described in ref. [9]. Oxides were prepared at three different growth rates: (i) linear potential ramping at $0.1 \mathrm{mV} / \mathrm{s}$, (ii) linear potential ramping at $1.0 \mathrm{mV} / \mathrm{s}$, and (iii) single-step polarization. The oxide growth voltages ranged from 1.5 to $6.5 \mathrm{~V}$ (SCE). This corresponds to an oxide thickness range of 4.5 to $19.5 \mathrm{~nm}$ based on the anodizing ratio of $3.0 \mathrm{~nm} / \mathrm{V}$ determined by Auger depth profile techniques $[9,12]$. In the rest of this paper, we will describe oxides in terms of their nominal thicknesses, determined from this ratio and the growth voltages. Oxides were grown at $0.1 \mathrm{mV} / \mathrm{s}$ to $2.5 \mathrm{~V}$ on two singlecrystal titanium substrates: one oriented in the (001) direction (Aremco) and the other having an orientation far from any low-index plane. No systematic differences between the photochemical spectra taken from oxides grown on the two kinds of substrates were observed.

Photoelectrochemical spectroscopy data were obtained as a function of applied potential and incident wavelength using illumination from a 450 W Xe arc lamp passed through a motor-driven grating monochromator (Oriel model 77250). A schematic of the system is given in fig. 1 . The 


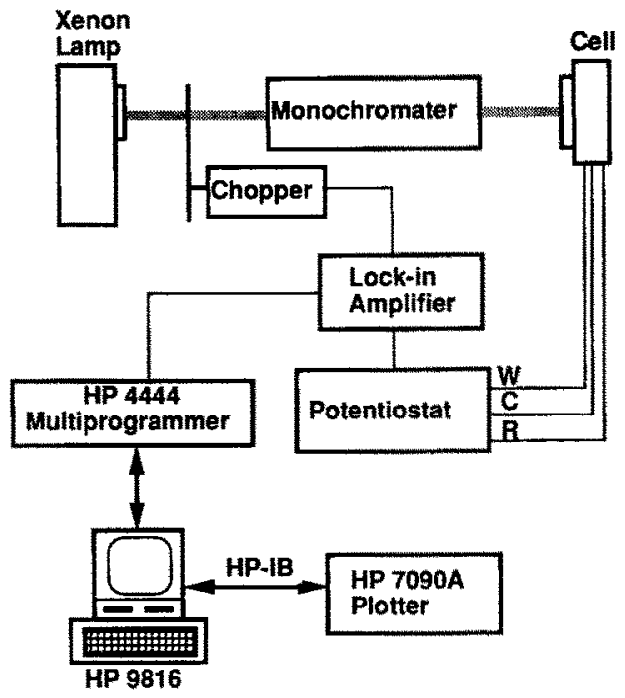

Fig. 1. Schematic of photoelectrochemical spectroscopy apparatus.

photoelectrochemical cell was constructed such that the impinging light passed through a $2 \mathrm{~mm}$ thick fused silica window and $5 \mathrm{~mm}$ of electrolyte before illuminating the specimen. A platinum wire was used as a counter electrode and an SCE as a reference electrode. The electrolyte was $0.05 \mathrm{M}$ $\mathrm{H}_{2} \mathrm{SO}_{4}$, the same electrolyte used for oxide growth. The electrochemical apparatus for control of the sample potential and for current measurement consisted of a PAR 173 potentiostat with a model 179 digital coulometer. In order to measure only the current due to illumination, lock-in detection techniques were used. The lamp output spectra was calibrated using a disk calorimeter (Scientech 36-0001) in order to convert the photocurrent signals to quantum efficiencies. Special care was taken to shield the calorimeter from heat so that the measured response was due to illumination only.

\section{Theoretical methods}

We calculate the density of states and the optical conductivity of models of the oxides using the equation of motion method [10]. (The relationship of the optical conductivity to the quantum ef- ficiency reported in the experiments is discussed below.) The basic approach of the equation of motion method is [13] to solve the time-dependent Schrödinger equation

$\mathrm{i} \hbar \partial \Psi / \partial t=H \Psi$,

and then to Fourier transform the result in time to obtain the quantities such as the density of states which are of interest at the end of the calculation. We work with a tight-binding Hamiltonian

$$
H=\sum_{i, \mu} \epsilon_{i, \mu} c_{i, \mu}^{\dagger} c_{i, \mu}+\sum_{i, \mu ; j, \psi}\left(t_{i, \mu ; j, \nu} c_{i, \mu}^{\dagger} c_{j, \nu}+\text { h.c. }\right)
$$

Here the is are site indices, the Greek letters $\mu, \nu$ are indices labelling orbitals and the sum on $(i j)$ is over neighbors on the lattice. We define a Green's function

$G_{i, \mu ;,, \mu}(t)=-\mathrm{i} \Theta(t)\left\langle\left\{c_{i, \mu}(t), c_{j, \nu}^{\dagger}(0)\right\}\right\rangle$

and an amplitude

$F_{i, \mu}(t)=\sum_{j, \nu} a_{j, \nu} G_{i, \mu ; j, \nu}(t)$.

The amplitudes $a_{j, y}$ can be chosen in various ways depending on what quantity is of particular interest. The equation of motion for $F_{i, \mu}$ is

$$
\mathrm{i} \hbar \partial F_{i, \mu} / \partial t=\sum_{j, \nu} H_{i, \mu ; j, \nu} F_{j, \nu},
$$

with initial condition

$F_{i, \mu}(0)=-i a_{i, \mu}$.

For example, we get the density of states $N_{\mu}(\omega)$ associated with the orbitals of type $\mu$ defined as

$N_{\mu}(\omega)=\sum_{n} \sum_{i}|\langle n \mid i, \mu\rangle|^{2} \delta\left(\omega-\epsilon_{n}\right)$,

by setting

$a_{i, \mu}=\mathrm{e}^{i \phi_{i, \mu}}$,

where $\phi_{i, \mu}$ is a number chosen at random from the interval $0<\phi_{i, \mu}<2 \pi$ for each site $i$ and for each orbital $\mu$ associated with that site. In these equations the ket $|i, \mu\rangle$ is the tight-binding state localized at site $i$ which is of orbital type $\mu$ and the states $|n\rangle$ are the eigenstates of the tight-binding 
problem in the (disordered) lattice. The density of states $N_{\mu}(\omega)$ is then

$N_{\mu}(\omega)=-\frac{1}{\pi} \operatorname{Im}\left[\int \sum_{i} \mathrm{e}^{-\mathrm{i} \phi_{i, \mu} F_{i, \mu}}(t) \mathrm{e}^{\mathrm{i} \omega t} \mathrm{~d} t\right]$.

Here the sum on $i$ extends only over sites with which an orbital of type $\mu$ is associated. For example, in the $\mathrm{TiO}_{2}$ problem, if $\mu$ is a p-orbital associated with the oxygen sites, then the sum extends only over the oxygen sites. The total density of states $N(\omega)$ is obtained by summing this quantity on orbital types $\mu$.

$N(\omega)=\sum_{\mu} N_{\mu}(\omega)$.

In principle, eq. (9) is only true if one averages the right-hand side over phases, meaning that it is only exact if we calculate the right-hand side many times, choosing different sets of random numbers to associate with each site and orbital each time, and then average the result. On the other hand, experience has shown that for large samples, just one set of randomly chosen phases gives a very accurate result. To get the local density of states defined as

$N_{i, \mu}(\omega)=\sum_{n}|\langle i, \mu \mid n\rangle|^{2} \delta\left(\omega-\epsilon_{n}\right)$,

we set

$a_{l, \nu}=-\mathrm{i} \delta_{l, v ; i, \mu}$,

where $i$ and $\mu$ are respectively the site and orbital of interest.

We discuss the numerical implementation of this method for calculating tight-binding densities of states in ref. [14] where we also discuss the relationship of the method to other sparse-matrix methods $[15,16]$.

To model rutile $\mathrm{TiO}_{2}$ we have used the full phenomenological tight-binding model of Vos [11] for which the parameters are summarized in ref. [10]. The model contains $5 \mathrm{~d}$-states per titanium site and 3 p-states and 1 s-state per oxygen site. Other details of the use of the equation of motion method for this model appear in ref. [10].

We have used these methods to calculate the electronic structure of systcms with substitutional disorder on the underlying rutile lattice, focusing in particular on random distributions of oxygen vacancies, which, because $\mathrm{TiO}_{2}$ is n-type, are expected to he the dominant point defect. We have not considered other types of disorder, such as grain boundaries or dislocations, in the work reported here. Neither have we considered the possibility that the oxygen vacancies might be ordered at some concentrations. In the rest of the paper, when we report theoretical calculations for a disordered model or system, we mean disordering in this sense that the system is disordered by a randomly placed set of oxygen vacancies. To represent an oxygen vacancy in the model, we use a Yukawa potential with a soft core, exactly as we did in refs. [10] and [14] where a discussion of the reasons for using this model appears. Specifically, for an oxygen vacancy at site $r_{O}$ we modify the diagonal matrix elements $\epsilon_{i, \mu}$ in eq. (2) by adding the quantity

$$
\frac{-Q \mathrm{e}^{-\beta\left|\boldsymbol{r}_{i}-\boldsymbol{r}_{\mathrm{o}}\right|}}{\epsilon_{\infty}\left|\boldsymbol{r}_{i}-\boldsymbol{r}_{\mathrm{O}}\right|}
$$

to each of them. Here $\boldsymbol{r}_{i}$ is the position of site $i$. We took $Q=2|e|, \epsilon_{\infty}=6.7$ and $\beta=5.0 \mathrm{~nm}^{-1}$. The values of $\epsilon_{i, \mu}$ at the oxygen vacancy sites were set to $2.0 \mathrm{eV}$. In the calculations which included oxygen vacancies, all the matrix elements $t_{t, \mu ; i, \nu}$ were left at the values used for the crystal without disorder as described above. Results for the density of states for rutile $\mathrm{TiO}_{2}$ with various numbers of randomly distributed oxygen vacancies were reported in ref. [10].

To relate the equation of motion calculations to the photocurrent data requires some further assumptions which we discuss next. We will assume that the quantum efficiency $\eta$ is proportional to the optical absorption coefficient of the sample. This assumption requires that the depletion layer width, $W$, and the minority carrier diffusion length, $L_{\mathrm{p}}$, are much smaller than the optical absorption distance, $1 / \alpha$. Capacitance studies of thin anodic $\mathrm{TiO}_{2}$ have shown that the donor distribution is uniform in anodic $\mathrm{TiO}_{2}$ oxides [5,17]. If we assume following Butler [6] that hole diffusion length is limited by bulk recombination then $L_{\mathrm{p}}=$ $\left(\epsilon k_{\mathrm{B}} T / 4 \pi e^{2} N_{\mathrm{d}}\right)^{1 / 2}$ in which we suppose that the mobility of electrons is much less than that of holes. With [17] $\epsilon=30$ and $N_{\mathrm{d}}=10^{20} / \mathrm{cm}^{3}$ we 
find $L_{\mathrm{p}} \approx 0.2 \mathrm{~nm}$. The depletion layer width $W_{0}$ at a potential of $1 \mathrm{~V}$ is estimated from [6] $W_{0}=$ $\left(2 \epsilon /|e| N_{\mathrm{d}}\right)^{1 / 2}$ from which we estimate using the same parameters that $W_{0} \approx 6 \mathrm{~nm}$. Finally, we estimate from data [18] on bulk samples that $1 / \alpha=10 \mathrm{~nm}$ for $\lambda=300 \mathrm{~nm}$ for $\mathrm{TiO}_{2}$ so that $L_{\mathrm{p}}<W<1 / \alpha$ for wavelengths above $300 \mathrm{~nm}$. Thus the assumption that the quantum efficiency is proportional to $\alpha$ is expected to be approximately valid in this region. In addition, for simplicity in applying our microscopic model, we have assumed that the effect of applying a potential to the oxide is a simple shift of the Fermi level. In terms of the same quantities discussed above, this requirement is that $1 / \alpha<W$ in contradiction with the requirement that $1 / \alpha>W$ in order that the current is proportional to $\alpha$. As one can see from our estimates, the quantities $1 / \alpha$ and $W$ are of the same order of magnitude in these experiments, so that both assumptions are in some question. We will return to this question in the discussion below.

With these assumptions we may calculate the spectral response of the photocurrent if we know the optical conductivity as a function of the Fermi energy. We obtain the optical conductivity from the densities of states which we calculate in the equation of motion method by use of the following simplified form of the Kubo-Greenwood formula [19]

$$
\begin{aligned}
\sigma(\omega)= & (\text { constant } / \omega) \times \int_{E_{F}-\hbar \omega}^{E_{F}} N(E) \\
& \times N(E+\hbar \omega) \mathrm{d} E
\end{aligned}
$$

$N(E)$ is the density of states at energy $E$ and $E_{\mathrm{F}}$ is the Fermi energy. This approximation assumes that the matrix elements coupling the light to the electron-hole pairs are independent of the wavevectors of the electron and hole created in the absorption process. This is certainly not correct, though it may be better for disordered oxides than for ordered ones as we will discuss below. Eq. (14) also ignores the fact that some of the states in the density of states of a disordered system are localized, leading to reduction of the conductivity for the localized states. To relate the optical conductivity to the quantum efficiency, we note that [20]

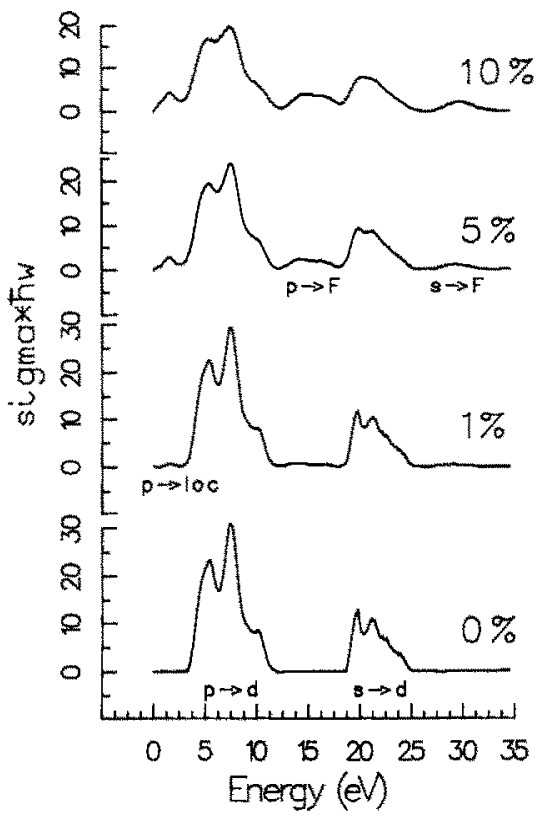

Fig. 2. Optical conductivity calculated using eq. (14) for the cases of $0,1,5$ and $10 \%$ oxygen vacancies in $\mathrm{TiO}_{2}$ in an $8 \times 8 \times 10$ unit cell "sample" with periodic boundary conditions in the directions normal to the $c$-axis.

$\alpha \propto \sigma / c$ (as long as the real part of the dielectric function is varying slowly with $\omega$ ) and that, as discussed above, we are assuming that $\eta \propto \alpha$. In fig. 2 we show the result of calculating $\omega \sigma(\omega)$ according to eq. (14) for the cases of $0,1,5$ and $10 \%$ oxygen vacancies and a "sample" which contains $8 \times 8 \times 10$ unit cells (3840 atomic sites) of $\mathrm{TiO}_{2}$ over a wide energy range. Periodic boundary conditions were imposed on the system in the directions normal to the $c$-axis. This model configuration approximates the situation in a $\mathrm{TiO}_{2}$ film about $3.0 \mathrm{~nm}$ thick.

In fig. 2 we have identified various transitions which are predicted to occur using these assumptions and our model. In the experiments reported here, only the region in the neighborhood of the bandgap around $3 \mathrm{eV}$ is relevant and we will show more detail from the calculation in that region below. As the oxygen vacancies are introduced into the model, one sees the development of bandgap states and transitions to and from them in the electron hole processes which arc described by $\omega \sigma(\omega)$ as shown in fig. 2 . 


\section{Results on slowly grown oxides}

The photoelectrochemical spectra of an anodic oxide grown to $5.5 \mathrm{~V}$ at $0.1 \mathrm{mV} / \mathrm{s}$ is shown in fig. 3. The photospectra shows a peak at a wavelength of about $280 \mathrm{~nm}$. Such a peak is typically observed for crystalline materials, including singlecrystal rutile [21]. Possible origins of the peak are discussed below. Here we focus on the spectral region near the band edge and on the low-energy side of the peak.

It has been traditional [6] to analyse the form of such spectra in the region near the bandgap using the assumption of parabolic bands near the band edge. Though we will show below that the assumptions on which it is based are not correct for the oxides under study, we will use the same form of analysis to facilitate comparison with earlier work. The assumption of parabolic bands, together with the assumption that the photocurrent is proportional to the absorption coefficient $\alpha$ leads to the relation

$\eta h \nu=\operatorname{const}\left(h \nu-E_{\mathrm{g}}\right)^{n}$,

where $\eta$ is the quantum efficiency and $n$ depends on whether the transition is direct, $n=\frac{1}{2}$, or indirect, $n=2$. If eq. (15) is true, then a plot of $(\eta h \nu)^{1 / n}$ will provide an $x$-axis intercept equal to the semiconductor bandgap. We only expect the assumptions on which eq. (15) is based to valid in

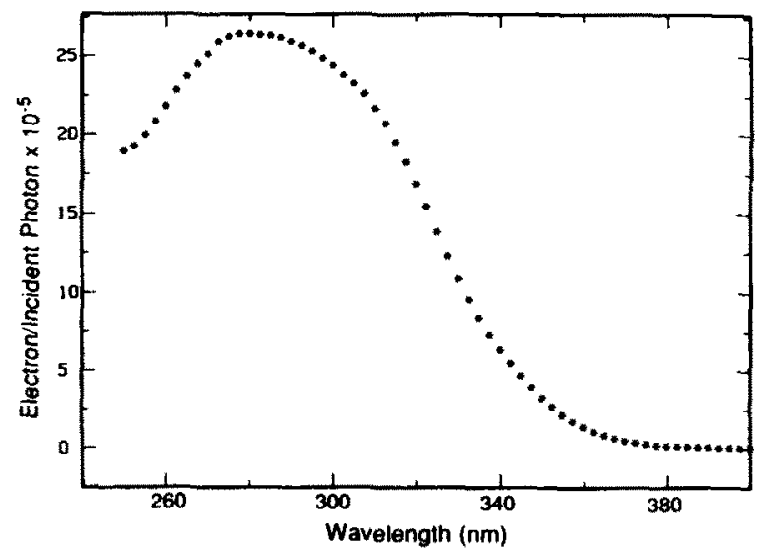

Fig. 3. Photospectra of an anodic oxide grown to $5.5 \mathrm{~V}$ on a polycrystalline titanium substrate by ramping the electrode potential at $0.1 \mathrm{mV} / \mathrm{s}$. Bias: $0.5 \mathrm{~V}$ (SCE).

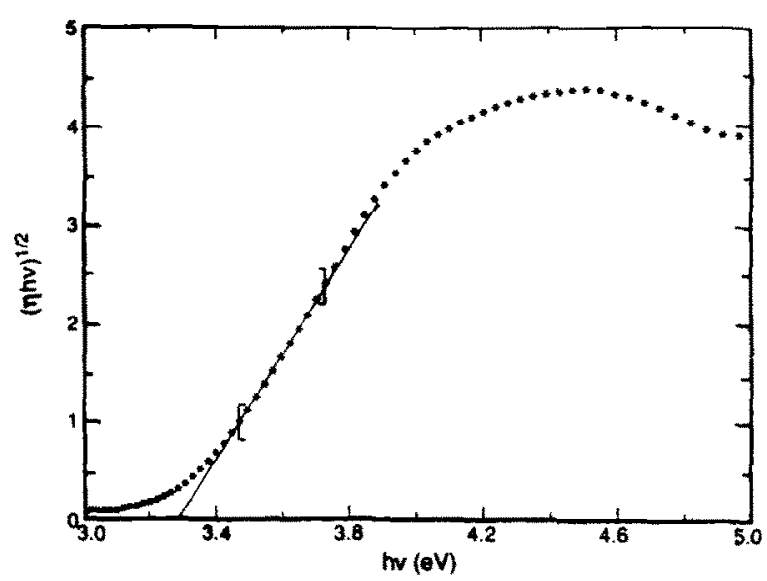

Fig. 4. $(\eta h v)^{1 / 2}$ versus $h v$ for data in fig. 3.

the limit of very good crystals and if the bands are parabolic in an energy region as large as the region in which the analysis is being applied. For the purposes of comparison with earlier work, we plot the data as $(\eta h \nu)^{1 / n}$ versus $h \nu$ as suggested by eq. (15).

Fig. 4 shows a plot of $(\eta h \nu)^{1 / 2}$ versus $h \nu$ for the data given in fig. 3 . The $x$-axis intercept might suggest an indirect bandgap of $3.3 \mathrm{eV}$. (The brackets, [ ], on the plots indicate the region used to obtain an intercept.) Fig. 5 is a plot of $(\eta h \nu)^{2}$ versus $h \nu$ for the same data. The linear region in this plot might suggest a direct bandgap of $3.7 \mathrm{eV}$. The region used for the second extrapolation does not overlap that of the first. Similar results were

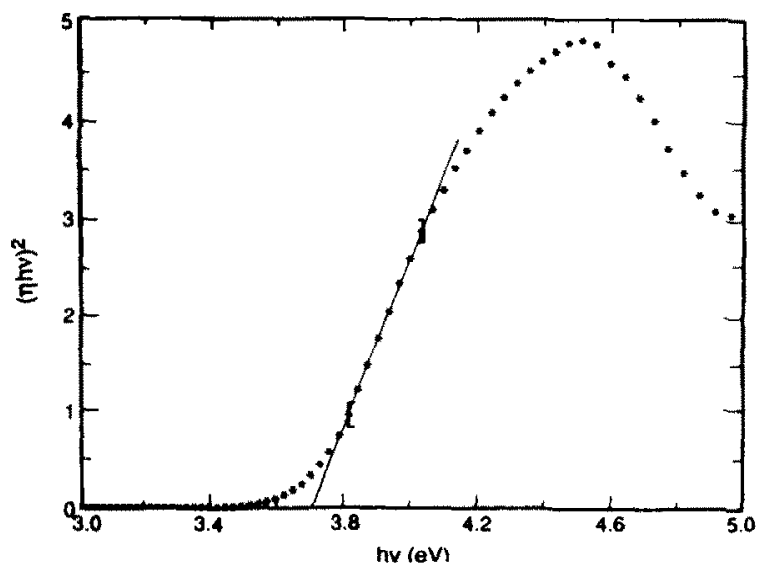

Fig. 5. $(\eta h \nu)^{2}$ versus $h \nu$ for data in fig. 3 . 
obtained in ref. [1] for anodic oxides grown to more than $17 \mathrm{~V}$.

We will now present evidence that the conclusion that the indirect bandgap in these oxides is of magnitude $3.3 \mathrm{eV}$ is spurious and that instead, the electronic structure suggested by the data of fig. 3 is very close to that of single-crystal rutile. The first evidence is obtained by a comparison of our data, plotted in the form of graphs of $(\eta h \nu)^{1 / 2}$ versus $h v$ and $(\eta h v)^{2}$ versus $h v$, respectively, with similar data from the photoelectrochemical spectra of single-crystal rutile as measured by Koffeyberg [22] as shown in fig. 6. It is clear that, in the region of energy associated with the plot of $(\eta h \nu)^{1 / 2}$ versus $h \nu$, the data are indistinguishable within experimental uncertainty. Thus the magnitude of the bandgap in this film and in singlecrystal rutile are essentially the same and equal to about $3.0 \mathrm{eV}$. This has not been noted in the literature before. The apparent indirect gap of 3.3 $\mathrm{eV}$ which is deduced by drawing a straight line through the data above $3.4 \mathrm{eV}$ and extending it down to the horizontal axis is not associated with a bandgap at all. (There are several possible transitions between bands at special points of the Brillouin zone which could account for a large increase in absorption around $3.3 \mathrm{eV}$. Perhaps the most likely candidate is the transition [11] $\Gamma_{s}^{+}(-9.541 \mathrm{eV}) \rightarrow R_{1}^{+}(-6.217 \mathrm{eV})$. To draw defi-

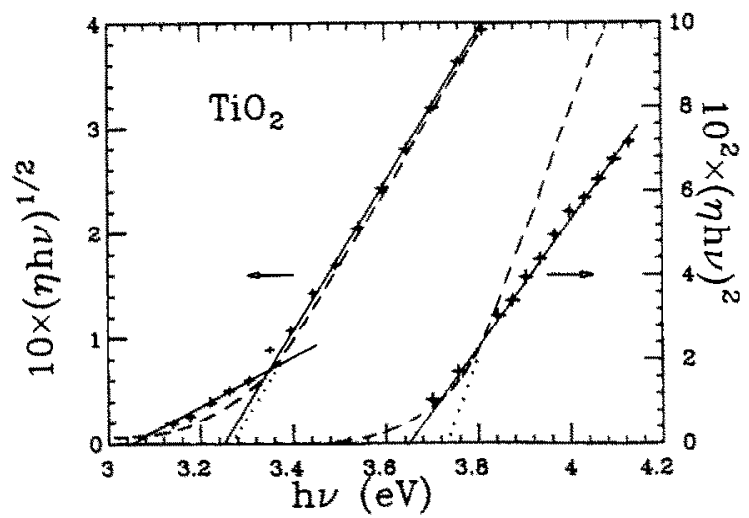

Fig. 6. Plots of $(\eta h v)^{1 / 2}$ (left scale) and $(\eta h \nu)^{2}$ (right scale) versus $h \nu$ for photocurrent data from single-crystal rutile $(+++)$ from Koffeyberg [22] and the anodic oxide grown at $0.1 \mathrm{mV} / \mathrm{s}(-\cdots)$.

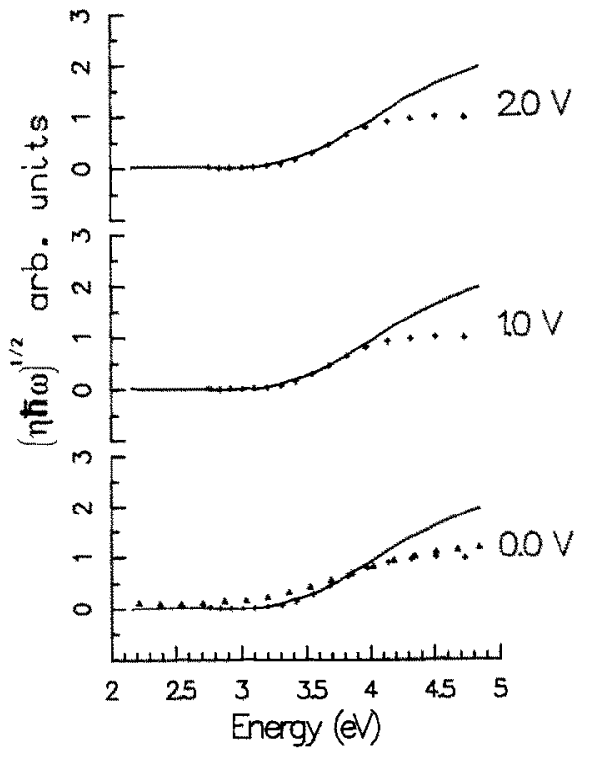

Fig. 7. Comparison of anodic photocurrent data (crosses) with equation of motion calculation (solid lines) of the optical conductivity for three potentials on the electrode. The vertical scale is arbitrary as discussed in the text. The triangles in the last figure show the results of the theoretical model simulation with $1 \%$ oxygen vacancies in the sample.

nitive conclusions would require a full group theoretic analysis which we have not undertaken.)

We make the point that there is no bandgap at $3.3 \mathrm{eV}$ in another way by comparing the results of our calculations of the photocurrent using the methods and assumptions described in section 3 with the observed photoresponse. While we have made several assumptions as discussed above in computing these results, we emphasize that we have not assumed that the bands are parabolic near the bandgap. Expressing the results as a plot of $(\eta h v)^{1 / 2}$ versus $h v$, we show the comparison in fig. 7 where, in the theoretical results, we modeled the oxide as a perfect layer of rutile $\mathrm{TiO}_{2}$ which was ten unit cells thick (as described at the end of the last section) and which contained no oxygen vacancies. In fig. 7 , one constant has been adjusted at each overpotential in order to set the vertical scale for the comparison of theory and experiment. Thus the agreement of the overall magnitude of the quantum efficiency in the theoretical and experimental curves has no significance. What is significant is the agreement be- 
tween theory and experiment with respect to the photon energy at which the photocurrent is predicted to become nonzero and the agreement between the shapes of the curves at photon energies just above the band edge. In these respects, the calculated spectra are very similar to those observed in the region near the band edge. The fact that near the bandgap at $3.0 \mathrm{eV}$ in this calculation the computed $\alpha$ is not proportional to $\left(\hbar \omega-E_{\mathrm{g}}\right)^{2}$ shows that the assumptions of parabolic bands implicit in eq. (15) is not valid here.

For comparison, we also show the results of a calculation of the optical conductivity in the case that $1 \%$ of the oxygen sites are vacant in fig. 7 as indicated by the triangles in the third part of the figure. The experimental results are in significantly worse agreement with the results of this calculation, suggesting that the spectral characteristics observed in this region of the spectrum are not caused by the presence of oxygen vacancies.

In fig. 7 one notes a discrepancy between the experimental and calculated spectra at higher photon energies. We think that this discrepancy is probably caused by breakdown in this frequency region of the condition that $1 / \alpha \gg W$ which is required in order that the photocurrent be proportional to the absorption coefficient. When this condition is violated, then a theory of the effects of a finite depletion layer thickness is required.

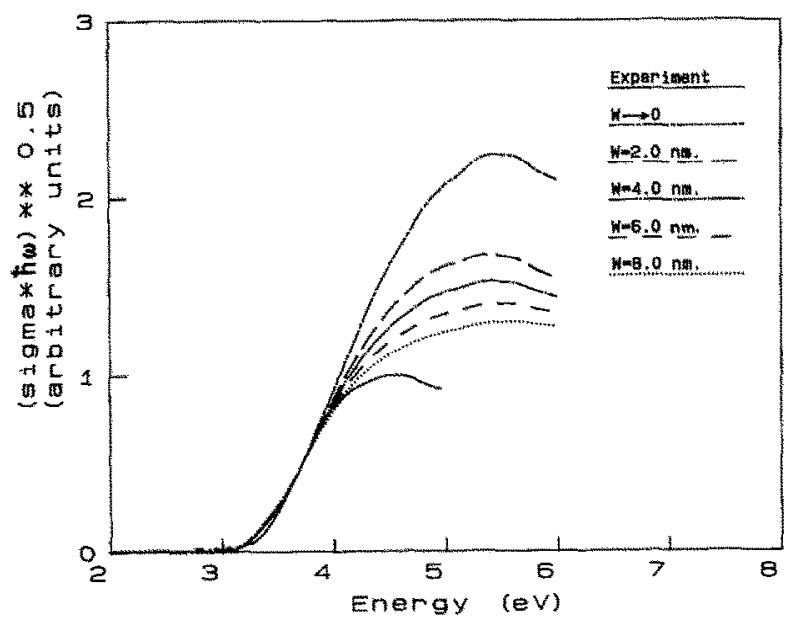

Fig. 8. Effects of the Butler theory of finite depletion thickness (eq. (16)) on the calculated currents.
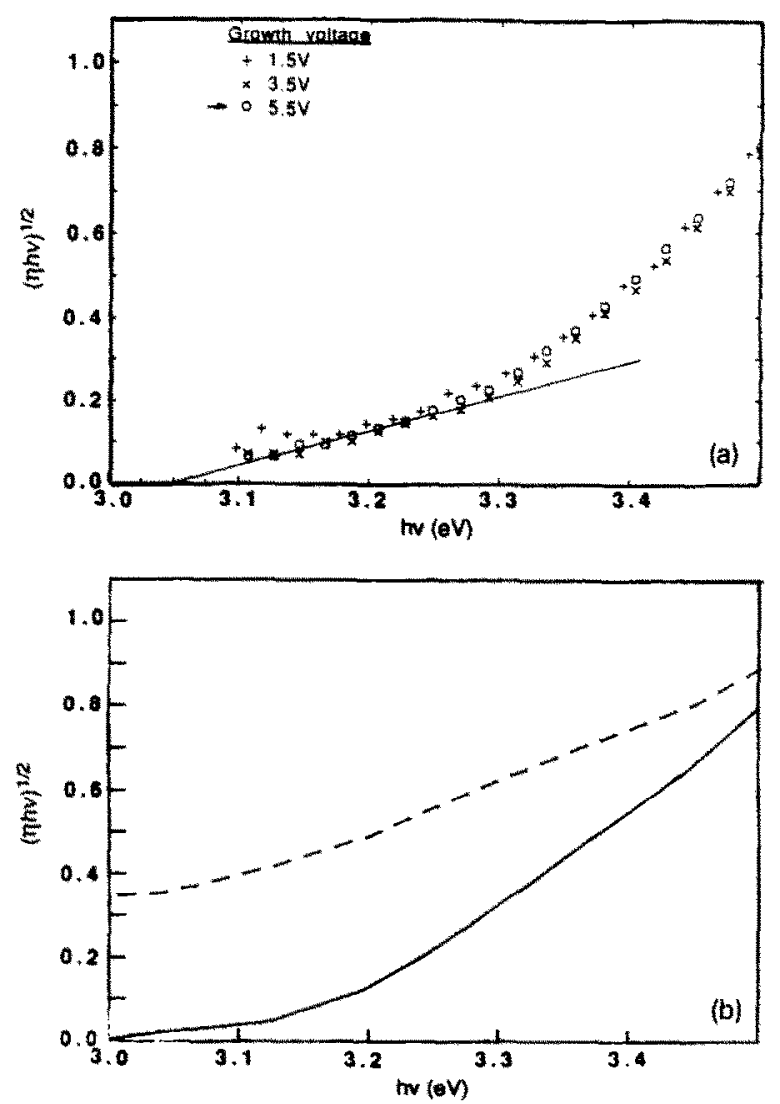

Fig. 9. (a) The near bandgap region of photospectra for oxides grown at $0.1 \mathrm{mV} / \mathrm{s}$ and to various final potentials (and hence various thicknesses) exhibited as $(\eta h \nu)^{1 / 2}$ versus $h v$. (b) Results of corresponding equation of motion calculations with 0 and 10 oxygen vacancies.

From the discussion in ref. [6], one obtains (when $\alpha L_{\mathrm{p}} \ll 1$ ), for example

$\eta \propto\left(1-\mathrm{e}^{-\alpha W}\right) / W$.

This has the effect of reducing the amplitude of the maximum in the photocurrent as shown in fig. 8 , where values of photocurrent (normalised to match experiment near the band edge) calculated using eq. (16) with various values of the depletion layer width are compared with the result of the experiment on the slowly grown oxide of thickness $7.5 \mathrm{~nm}$. One sees that the amplitude of the peak above the band edge is reduced by the correction for finite depletion layer width in eq. (16), bring- 
ing theory and experiment into better agreement with regard to the height of the peak. However, the position of the peak is not accurately predicted by eq. (16).

Next we show results for the photospectra of three slowly grown oxides grown to different potentials, and correspondingly having different thicknesses in fig. 9a. The results are plotted as $(\eta h \nu)^{1 / 2}$ versus $h \nu$ near the gap energy. One sees a similar behavior for the lower voltage (and hence thinner) oxides except that there may be a suggestion of some additional structure just above the gap for the thinnest film grown to $4.5 \mathrm{~nm}(1.5 \mathrm{~V}$ (SCE)).

Evidence that the thinnest slowly grown oxide was behaving differently came from a study of the photocurrent as a function of potential at fixed light wavelength. The traditional [23] Schottky barrier model, assuming constant charge density in the depletion region and no localized states, together with the analysis of the photocurrent given by Butler [6] predicts that the photocurrent should be $\propto\left(V-V_{\mathrm{fb}}\right)^{1 / 2}$ where $V_{\mathrm{fb}}$ is the flat band potential. In fig. 10 we show plots of the photocurrent squared versus $V$ for two oxides and two wavelengths. The simple prediction is seen in the thicker oxide but not in the thin one, grown to $1.5 \mathrm{~V}$ (SCE) and about $4.5 \mathrm{~nm}$ thick. For that

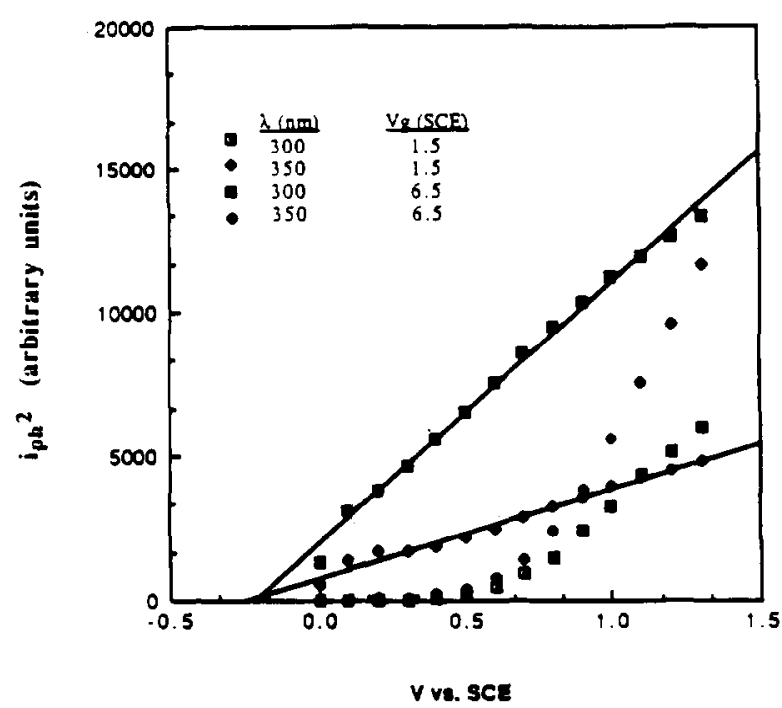

Fig. 10. Photocurrent squared versus potential at two fixed wavelengths for two oxides.

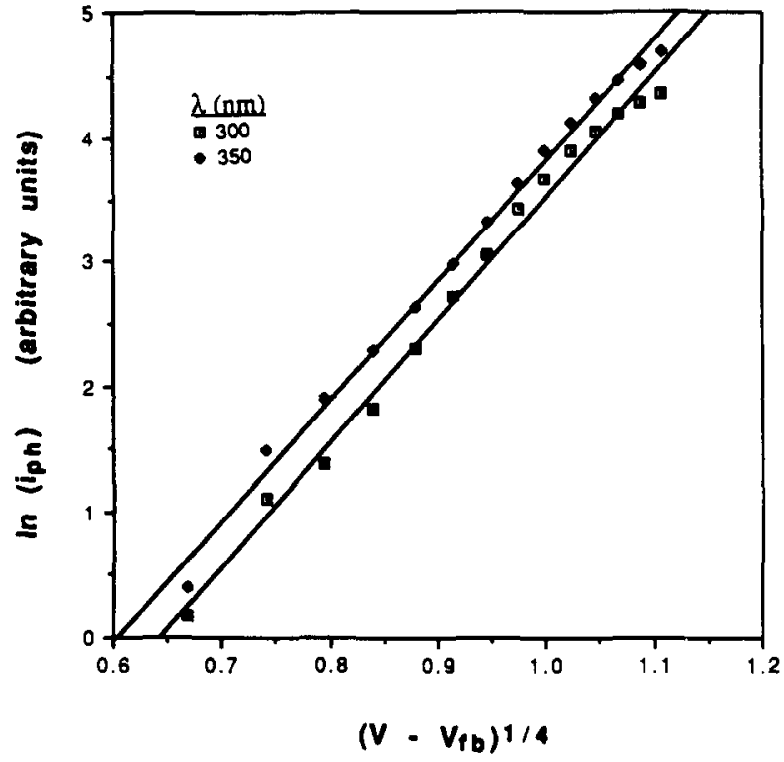

Fig. 11. In $i$ versus $\left(V-V_{\mathrm{fb}}\right)^{1 / 4}$ for the sample oxide grown slowly to $1.5 \mathrm{~V}$ (SCE).

sample, the photocurrent as a function of potential behaves more as predicted for current from localized electronic states [24]

$\ln i \propto\left(V-V_{\mathrm{fb}}\right)^{1 / 4}$,

as shown in fig. 11.

We also noted in fig. 9a that the observed photocurrent for the thin $(4.5 \mathrm{~nm})$ slowly grown oxide might have some additional structure at frequencies just above the bandgap. Though the experimental reproducibility of this structure has not been checked and is open to doubt, we explored whether such structure is to be expected from a film with a significant density of oxygen vacancies. We plotted our calculated photoresponse on the same energy scale as shown in fig. $9 \mathrm{~b}$. In fig. $9 \mathrm{~b}$ the solid line shows the calculated spectrum for a model without oxygen vacancies while the dashed curve shows the result with $1 \%$ of the oxygen sites vacant. The defects are predicted to increase the photocurrent in this spectral range. (However capacitance measurements indicate a vacancy concentration in these oxides of $10^{18}$ to $10^{20} \mathrm{~cm}^{-3}$ which is substantially less than $1 \%$ of oxygen sites $\left(6.4 \times 10^{20} \mathrm{~cm}^{-3}\right)$.) We note that the calculated increase is not a completely obvious 


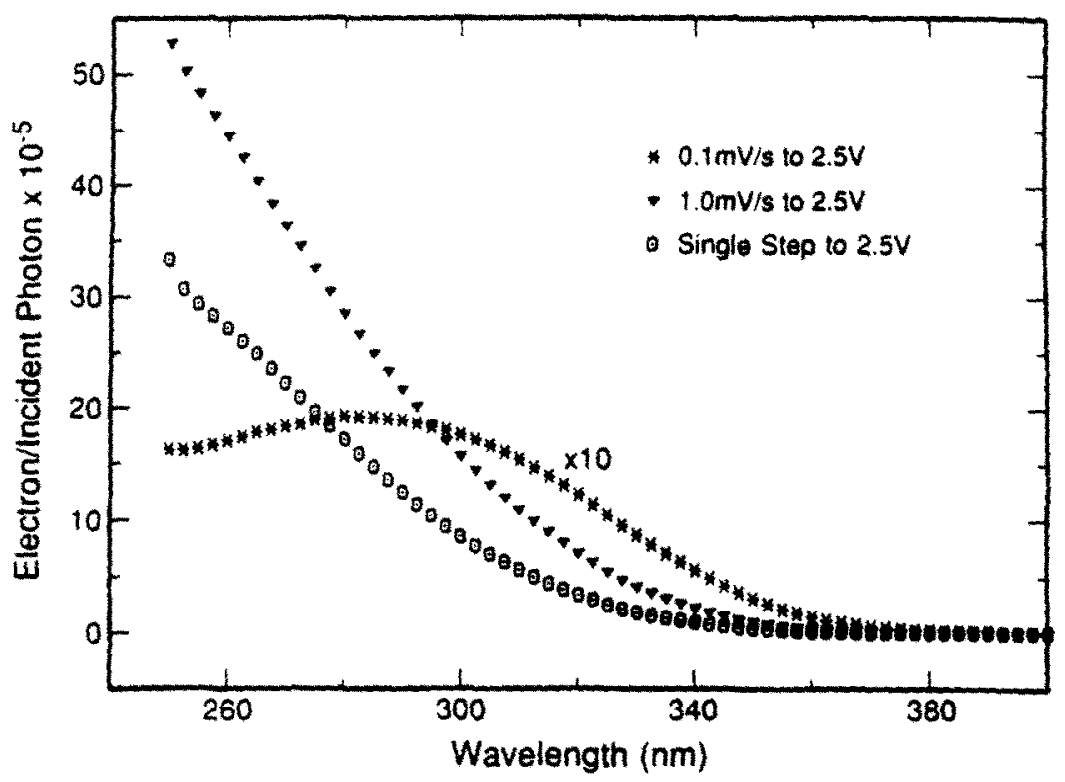

Fig. 12. Photocurrent spectra for $\mathrm{TiO}_{2}$ oxides grown to $2.5 \mathrm{~V}$ at different rates; electrode bias: $1.0 \mathrm{~V}$ (SCE).

result because this spectral range is above the band edge. A more detailed exploration showed that the spectral weight gained in this region when defects are added is compensated by a decrease, as it must be, at higher frequencies.

\section{Results on rapidly grown oxides}

Electron diffraction studies showed that as the oxide growth rate is increased from potential ramping at $0.1 \mathrm{mV} / \mathrm{s}$ to single-step polarization, the oxide becomes more structurally disordered and the oxide grain size decreases. Fig. 12 shows photospectra of three oxides grown to $2.5 \mathrm{~V}$ at different rates on polycrystalline titanium substrates. The oxides grown by single-step polarization or by ramping at $1.0 \mathrm{mV} / \mathrm{s}$ produced similar spectra which did not include the peak observed for the $0.1 \mathrm{mV} / \mathrm{s}$ oxides. Notice that the photocurrents are a factor of 10 smaller in these more structurally disordered oxides. Fig. 13a shows plots of $(\eta h v)^{1 / 2}$ versus $h \nu$ for the data on the three oxides from which the data in fig. 12 were $o b$ tained. The intercepts of the linear regions with the $x$-axis are similar for all three oxides, but shape is very different for the rapidly grown ones.
In particular, $\eta h \nu$ is proportional to $v^{2}$ over most of the range of the plot for the more disordered oxides. A plot of $(\eta h \nu)^{2}$ versus $h \nu$ (fig. 13b) shows that there is no linear region for the rapidly grown oxides, making any determination of a "direct gap" impossible.

In view of the fact that the electron diffraction studies showed that the rapidly grown oxides consisted of small crystallites $(-5 \mathrm{~nm}$ in size) of rutile we believe that these results may arise from matrix element effects in the optical absorption coefficient as follows: The overall decrease in the photocurrent amplitude may be attributable to a large number of localized states acting as recombination centers at the surfaces of crystallites and in the grain boundaries between them into which light is absorbed without contributing to the photocurrent. Within the crystallites, finite size effects may accentuate the breaking of wavevector conservation in the electron-hole pair creation process, so that the direct gap (momentum conserving) process is supressed relative to the indirect gap (momentum nonconserving) process. (The lower amplitude of the photocurrent in the rapidly grown samples may also account for the absence of a peak around $4.4 \mathrm{eV}$ in the photospectra in fig. 12.) In the case of the rapidly grown oxides, the 

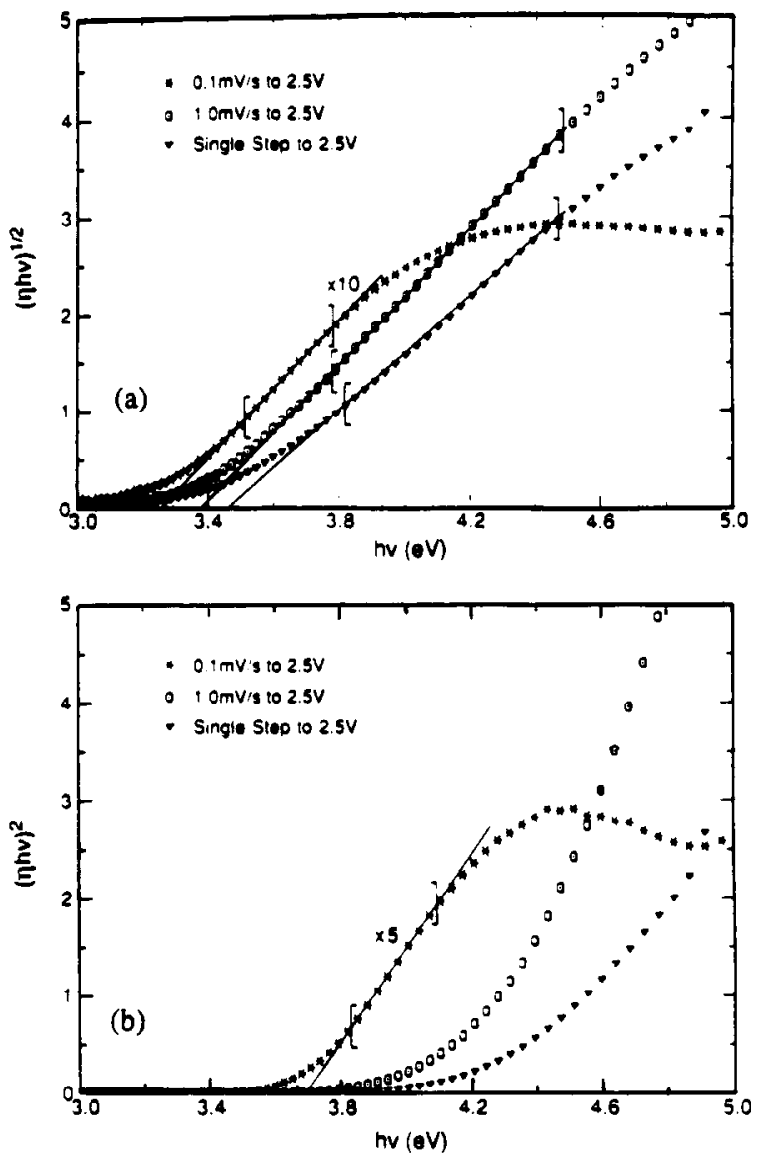

Fig. 13. (a) $(\eta h \nu)^{1 / 2}$ versus $h \nu$ for the data of fig. 12 . (b) $(\eta h \nu)^{2}$ versus $h \nu$ for the same data.

magnitude of the current is much smaller, suggesting that the relevant part of the absorption coefficient is smaller and the assumption $1 / \alpha \gg W$ is more likely to be valid. As discussed above, omitting finite depletion layer thickness corrections causes the calculated currents to rise more steeply with photon frequency at frequencies above $4 \mathrm{eV}$, as observed in these rapidly grown oxides.

\section{Conclusions and discussion}

On slowly grown $(0.1 \mathrm{mV} / \mathrm{s})$ oxides thicker than $10 \mathrm{~nm}$ we have shown that the electronic structure of oxides on titanium as revealed by photoelectrochemical spectroscopy is very similar to that of bulk rutile. We showed this both by direct comparison with experiments on bulk rutile and by comparisons of the electrochemical results with the results of detailed electronic structure calculations. Earlier work that attributed photoconductivity near the band edge to gap states or an Urbach tail implicitly and incorrectly assumed that the band structure of rutile near the band edge could be described by a single-parabolic band.

Our result is somewhat surprising because it is very likely that there are enough vacancies in the slowly grown oxides to produce an observable number of gap states. A possible explanation is that, though gap states exist, they do not contribute to the photoconductivity because they are localized. To check this assumption theoretically we require a better calculation of the optical conductivity than that given by eq. (14) which, as we pointed out, neglects the effects of localization. Very recently [25] we have performed calculations which include localization effects on a simplified model of the oxide. The new calculations, which will be reported in more detail elsewhere, suggest that the gap states arising from vacancies may not contribute to the photoconductivity.

The calculation reported here contains the effects of a surface potential in the sense that periodic boundary conditions are employed in only two of the three pairs of faces of the computational sample. We have shown, however, that the surface potential has a negligible effect on the density of states of this model by an explicitly calculation of the surface density of states. Our calculational results do not take account of the depletion layer, because they are not electrostatically self-consistent. This should not matter as long as $1 / \alpha \gg W$. As we have discussed above, for some of the experiments, it is likely that $1 / \alpha$ $\approx W$ and in those cases, an electrostatically selfconsistent calculation is required. Results of such self-consistent calculations will be described elsewhere.

On very thin $(\sim 4.5 \mathrm{~nm})$ slowly grown oxides and on more rapidly grown oxides, the photospectra reveal a different electronic structure. The thin, slowly grown oxides have spectra like those expected for bulk rutile with large numbers of point defects. The rapidly grown oxides have spectra 
and morphology which suggest very small crystallites of rutile.

\section{Acknowledgements}

This work was supported in part by the Department of Energy Office of Basic Energy Sciences through the Corrosion Research Center (grant DE-FG02-88ER45338) and by the Minnesota Supercomputer Institute. Chris McMillan and Jim Kakalios are thanked for reading the manuscript and providing helpful suggestions.

\section{References}

[1] K. Leitner, J.W. Schultze and U. Stimming, J. Electrochem. Soc. 133 (1986) 1561.

[2] J.W. Schultze, U. Stimming and J. Weise, Ber. Bunsenges. Phys. Chem. 86 (1972) 276.

[3] C.E. Vallet, S.E. Borns and J.S. Hendreikson, J. Electrochem. Soc. 135 (1988) 387.

[4] J.C. Pesant and P. Vennereau, J. Electroanal. Chem. 106 (1980) 103.

[5] J.F. McAleer and L.M. Peter, Faraday Disc. Chem. Soc. 70 (1980) 67.

[6] W.W. Gaertner, Phys. Rev. 116 (1959) 84; M.A. Butler, J. Appl. Phys. 48 (1977) 1914.

[7] R. Tauc, in: Optical Properties of Solids, Ed. F. Abeles (North-Holland, Amsterdam, 1972).
[8] P.A. Martin, B. Streetman and K. Hess, J. App. Phys. 52 (1981) 7409.

[9] M.R. Kozlowski, P.S. Tyler, W.H. Smyrl and R.T. Atanasoski, Surf. Sci. 194 (1988) 505.

[10] J.W. Halley, M.T. Michalewicz and N. Tit, Phys. Rev. B 41 (1990) 10165.

[11] K. Vos, J. Phys. C 10 (1977) 3917.

[12] M.R. Kozlowski, W.H. Smyrl, Lj. Atanasoska and R.T. Atanasoski, Electrochim. Acta 34 (1989) 1763.

[13] R. Alben, M. Blume, H. Krakauer and L. Schwartz, Phys. Rev. B 12 (1975) 4090.

[14] J.W. Halley and H. Shore, Phys. Rev. B 36 (1987) 6640.

[15] R. Haydock, V. Heine and M.J. Kelly, J. Phys. C 5 (1972) 2845.

[16] D. Weaire and E.P. O'Reilly, J. Phys. C 18 (1985) 1401.

[17] R.M. Torresi, O.R. Camara and C.P. DePauli, Electrochim. Acta. 32 (1987) 1291.

[18] F. Mollers, H. Tolle and R. Memming, J. Electrochem. Soc. 121 (1974) 1160.

[19] N.F. Mott and E.A. Davis, Electronic Processes in NonCrystalline Materials (Clarendon Press, Oxford, 1971) p. 10.

[20] J.D. Jackson, Classical Electrodynamics, 2nd ed. (Wiley, New York, 1975) p. 286.

[21] L.A. Harris and R. Wilson, J. Electrochem. Soc. 123 (1976) 1010.

[22] P. Koffeyberg, K. Dwight and A. Wold, Solid State Commun. 30 (1979) 433.

[23] J. Bardeen, Phys. Rev. 71 (1947) 717.

[24] P.A. Martin, B. Streetman and K. Hess, J. Appl. Phys. 52 (1981) 7409.

[25] N. Tit, J.W. Halley, M.T. Michalewicz and H.B. Shore, Bull. Am. Phys. Soc. 36 (1991) 976. 